\title{
Estimates of Dirichlet Eigenvalues for One-Dimensional Fractal Drums
}

\author{
Hua Chen* and Jinning Li \\ School of Mathematics and Statistics, Wuhan University, Wuhan, Hubei 430072, \\ China \\ Received 11 November 2019; Accepted (in revised version) 2 December 2019 \\ Dedicated to Professor Weiyi Su on the occasion of her 80th birthday
}

\begin{abstract}
Let $\Omega$, with finite Lebesgue measure $|\Omega|$, be a non-empty open subset of $\mathbb{R}$, and $\Omega=\bigcup_{j=1}^{\infty} \Omega_{j}$, where the open sets $\Omega_{j}$ are pairwise disjoint and the boundary $\Gamma=\partial \Omega$ has Minkowski dimension $D \in(0,1)$. In this paper we study the Dirichlet eigenvalues problem on the domain $\Omega$ and give the exact second asymptotic term for the eigenvalues, which is related to the Minkowski dimension $D$. Meanwhile, we give sharp lower bound estimates for Dirichlet eigenvalues for such one-dimensional fractal domains.
\end{abstract}

Key Words: One-dimensional fractal drum, Dirichlet eigenvalues, Pólya conjecture, Minkowski dimension.

AMS Subject Classifications: 52B10, 65D18, 68U05, 68U07

\section{Introduction and main results}

Let $\Omega$, with boundary $\Gamma=\partial \Omega$ be a non-empty open subset of $\mathbb{R}^{n}(n \geq 1)$. We assume that $\Omega$ has finite Lebesgue measure $|\Omega|$. Then we consider the following Dirichlet eigenvalues problem:

$$
\begin{cases}-\Delta u=\lambda u & \text { in } \Omega \\ u=0 & \text { on } \Gamma .\end{cases}
$$

As is well-known (or see [4]), the problem (1.1) has a sequence of discrete eigenvalues, which can be ordered, after counting finite multiplicity as

$$
0<\lambda_{1} \leq \lambda_{2} \leq \cdots \leq \lambda_{k} \leq \cdots \quad \text { and } \quad \lambda_{k} \rightarrow+\infty \quad \text { as } k \rightarrow+\infty
$$

${ }^{*}$ Corresponding author. Email addresses: chenhua@whu.edu.cn (H. Chen), lijinning@whu.edu.cn (J. N. Li) 
In 1911, Weyl studied the problem (1.1) and obtained the following first asymptotic term for a bounded open $\Omega$ in $\mathbb{R}^{n}$ (see $[16,17]$ ):

$$
\lambda_{k} \sim \frac{4 \pi^{2} k^{\frac{2}{n}}}{\left(B_{n}|\Omega|\right)^{\frac{2}{n}}} \quad \text { as } \quad k \rightarrow+\infty,
$$

where $B_{n}$ is the volume of the unit ball in $\mathbb{R}^{n}$ and we say that $f_{k} \sim g_{k}$ as $k \rightarrow+\infty$ means $\frac{f_{k}}{g_{k}} \rightarrow 1$ as $k \rightarrow+\infty$.

In 1961, Pólya [14] gave his conjecture for any bounded open subset $\Omega$ in $\mathbb{R}^{n}$, the Dirichlet eigenvalues has the following lower bounds for any $k \geq 1$,

$$
\lambda_{k} \geq \frac{4 \pi^{2} k^{\frac{2}{n}}}{\left(B_{n}|\Omega|\right)^{\frac{2}{n}}}
$$

also he proved his conjecture (1.3) would be true when $\Omega$ is a plane domain which tiles $\mathbb{R}^{2}$. Later in 1983, Peter Li and Yau [12] proved for general bounded domain $\Omega$ with smooth boundary, the Dirichlet eigenvalues had the following lower bounds:

$$
\sum_{i=1}^{k} \lambda_{i} \geq \frac{n}{n+2} \frac{4 \pi^{2} k^{\frac{2+n}{n}}}{\left(B_{n}|\Omega|\right)^{\frac{2}{n}}} \quad \text { for any } \quad k \geq 1 .
$$

In this direction, there are a lot of research works on eigenvalues for smooth domains (or regular domains), e.g., one can see $[7,8,13,15]$.

However, when $\Omega$ is fractal domain, i.e., its boundary $\Gamma$ is "fractal", the situation will be more complicated (cf. [2,3,5,9-11]). Here in this paper, we shall study the case for $\Omega$ is one-dimensional fractal string (see the definition below). We shall give the results for second asymptotic term and precise lower bound estimates of Dirichlet eigenvalues for such kinds of fractal sets.

Let us start to consider the open bounded subset $\Omega \in \mathbb{R}^{n}$ with boundary $\Gamma$, we shall first give some definitions about Minkowski measurability and Minkowski dimension as followes:

Definition 1.1. Let $\Gamma_{\varepsilon}=\{x: d(x, \Gamma)<\varepsilon\}$, where $d(x, \Gamma)$ denotes the Euclidean distance of $x$ to the boundary $\Gamma$. For $d \in[n-1, n]$, the (d-dimensional) upper Minkowski content and lower Minkowski content of $\Gamma$ are given by

$$
\mathcal{M}^{*}(d ; \Gamma) \triangleq \limsup _{\varepsilon \rightarrow 0^{+}} \varepsilon^{-(n-d)}\left|\Gamma_{\varepsilon} \cap \Omega\right|, \quad \mathcal{M}_{*}(d ; \Gamma) \triangleq \liminf _{\varepsilon \rightarrow 0^{+}} \varepsilon^{-(n-d)}\left|\Gamma_{\varepsilon} \cap \Omega\right| .
$$

The Minkowski dimension of $\Gamma$ is defined as

$$
D=\inf _{d \in[n-1, n]}\left\{d: \mathcal{M}^{*}(d ; \Gamma)<+\infty\right\} .
$$

If $0<\mathcal{M}_{*}(D ; \Gamma)=\mathcal{M}^{*}(D ; \Gamma)=\mathcal{M}(D ; \Gamma)<+\infty$, then $\Gamma$ is $D$-dimensional Minkowski measurable with Minkowski measure $\mathcal{M}(D ; \Gamma)$. 
In case of $D \in(n-1, n]$, we call $\Omega$ the domain with fratcal boundary $\Gamma$ (or called the fractal domain). If $n=1$, we have the following definition:

Definition 1.2 (Fractal string). Let

$$
\Omega=\bigcup_{j=1}^{\infty} \Omega_{j}
$$

be a non-empty open subset of $\mathbb{R}$, with finite Lebesgue measure

$$
|\Omega|=\sum_{j=1}^{\infty}\left|\Omega_{j}\right|<+\infty
$$

where the open sets $\Omega_{j}$ are pairwise disjoint. Let $\ell_{j} \triangleq\left|\Omega_{j}\right|$, and set $\ell_{1}, \cdots$ from large to small. If Minkowski dimension for the boundary of $\Omega D \in(0,1]$, we call $\mathcal{L}=\left\{\ell_{j}\right\}_{j=1}^{\infty}$ the fractal string of $\Omega$.

Example 1.1. Let $\Omega=\bigcup_{j=1}^{\infty} \Omega_{j}, \Omega_{j}$ are pairwise disjoint intervals, and $\left|\Omega_{j}\right|=\ell_{j}, \sum_{j=1}^{+\infty} \ell_{j}<$ $+\infty$. Denote $\mathcal{L}=\left\{\ell_{j}\right\}_{j=1}^{\infty}$ as the fractal string of $\Omega$, in each $\Omega_{j}$ there exists a local eigenvalues

$$
\lambda_{m, j}\left(\Omega_{j}\right)=\frac{\pi^{2} m^{2}}{\ell_{j}^{2}} \text { for } m, j=1, \cdots .
$$

Then we extend their eigenfunctions $u_{m, j}$ to be zero on other intervals, so we obtain the expression of $\lambda_{m, j}$ in the whole $\Omega$ :

$$
\lambda_{m, j}=\frac{\pi^{2} m^{2}}{\ell_{j}^{2}} \text { for } m, j=1, \cdots .
$$

After arranging them from small to large, we get the all eigenvalues of (1.1).

In the case of $n=1$ and $D \in(0,1)$, Lapidus [9] proved that if $\mathcal{L}=\left\{\ell_{j}\right\}_{j=1}^{\infty}$ is the fractal string of $\Omega$. Then the condition $\ell_{j} \asymp j^{-\frac{1}{D}}$ as $j \rightarrow+\infty$ holds, (where $f(x) \asymp g(x)$ (as $x \rightarrow+\infty$ ) means that there exists $X>0$ and $0<a \leq b<+\infty$ such that when $x>X, a f(x) \leq g(x) \leq b f(x))$, iff the boundary $\Gamma$ of $\Omega$ has Minkowski dimension $D$ and $0<\mathcal{M}_{*}(D ; \Gamma) \leq \mathcal{M}^{*}(D ; \Gamma)<+\infty$. In this case, Lapidus [9] in 1993 gave that

$$
\varphi(\lambda)-N(\lambda) \asymp \lambda^{\frac{D}{2}} \text { for } \lambda \rightarrow+\infty,
$$

where

$$
N(\lambda)=\sharp\left\{k \geq 1: \lambda_{k} \leq \lambda\right\}
$$

is Dirichlet counting function,

$$
\varphi(\lambda)=|\Omega| \frac{\sqrt{\lambda}}{\pi}
$$


is called "Weyl term".

Furthermore, if there exists $L>0$ then the condition $\ell_{j} \sim L j^{-\frac{1}{D}}$ as $j \rightarrow \infty$ holds iff the boundary $\Gamma$ is $D$-dimensional Minkowski measurable and

$$
\mathcal{M}(D ; \Gamma)=\frac{2^{1-D}}{1-D} L^{D}
$$

In this case, when the boundary is Minkowski measurable, Lapidus [9] proved the following result for second asymptotic term of Dirichlet counting function $N(\lambda)$ :

$$
N(\lambda)=\varphi(\lambda)-c_{D} \mathcal{M}(D ; \Gamma) \lambda^{\frac{D}{2}}+o\left(\lambda^{\frac{D}{2}}\right) \text { for } \lambda \rightarrow+\infty,
$$

where $c_{D}=2^{-(1-D)} \pi^{-D}(D-1) \zeta(D)(\zeta(z)$ is Riemann-zeta function). In this paper, by using Lapidus' result, we shall give the exact second asymptotic term of Dirichlet eigenvalues $\lambda_{k}$, namely

Theorem 1.1 (Asymptotic expansion). Let $\Omega=\cup_{j=1}^{\infty} \Omega_{j}$ be a non-empty open subset of $\mathbb{R}$, with finite Lebesgue measure $|\Omega|=\sum_{j=1}^{\infty}\left|\Omega_{j}\right|<+\infty$, where the open sets $\Omega_{j}$ are pairwise disjoint. Let $\ell_{j} \triangleq\left|\Omega_{j}\right|$, and $\mathcal{L}=\left\{\ell_{j}\right\}_{j=1}^{\infty}$ is the fractal string of $\Omega$. The boundary $\Gamma$ of $\Omega$ has Minkowski dimension $D \in(0,1)$, and $\left\{\lambda_{k}\right\}_{k=1}^{\infty}$ are the Dirichlet eigenvalues of (1.1). Then

$$
\lambda_{k}-\frac{\pi^{2}}{|\Omega|^{2}} k^{2} \asymp k^{1+D} \quad \text { as } \quad k \rightarrow+\infty .
$$

Furthermore, if $\ell_{j} \sim L j^{-\frac{1}{D}},(j \rightarrow \infty)$ for some constant $L>0$, then

$$
\lambda_{k}=\frac{\pi^{2}}{|\Omega|^{2}} k^{2}+\frac{\mathcal{T}_{D} \mathcal{M}(D ; \Gamma)}{|\Omega|^{2+D}} k^{1+D}+o\left(k^{1+D}\right) \text { as } k \rightarrow+\infty,
$$

where $\mathcal{M}(D ; \Gamma)$ is Minkowski measure of $\Gamma$, the constant $\mathcal{T}_{D}=-2^{D} \zeta(D)(1-D) \pi^{2}>0$ and $\zeta(z)$ is Riemann-zeta function.

Theorem 1.1 also gives an estimate of the multiplicity of the eigenvalues, see Subsection 2.3 below.

Let's turn to the second result, the lower bounds for the Dirichlet eigenvalues. For one-dimensional fratcal domain, if its boundary has Minkowski dimension $D \in(0,1)$, then we know that $\ell_{j} \asymp j^{-\frac{1}{D}},(j \rightarrow \infty)$, i.e., there exist $0<\alpha_{*} \leq \alpha^{*}<+\infty$ such that for all $\ell_{j}$ which will satisfy

$$
0<\alpha_{*} \leq L_{j} \leq \alpha^{*}<+\infty \text { for } j \geq 1, \quad L_{j}=\ell_{j} j^{\frac{1}{D}} .
$$

Now our second result will be given as follows: 
Theorem 1.2 (Lower bounds of eigenvalues). Under the conditions in Theorem 1.1, if the boundary $\Gamma$ has Minkowski dimension $D \in(0,1)$, then there exist positive constants $\alpha_{*}$ and $\alpha^{*}$ (as defined by (1.11)). such that the Dirichlet eigenvalues $\left\{\lambda_{k}\right\}_{k=1}^{\infty}$ of (1.1) have the following lower bounds:

$$
|\Omega| \frac{\sqrt{\lambda_{k}}}{\pi} \geq k+\frac{P}{|\Omega|^{D}} k^{D} \quad \text { for } \quad k \geq 1 .
$$

More precise, for any $k \geq 1$ we have

$$
\lambda_{k} \geq \frac{\pi^{2}}{|\Omega|^{2}} k^{2}+\frac{2 \pi^{2} P}{|\Omega|^{2+D}} k^{1+D}+\frac{\pi^{2} P^{2}}{|\Omega|^{2+2 D}} k^{2 D}
$$

where

$$
P=\frac{D \alpha_{*}}{\left(\alpha^{* D}+\ell_{1}^{D}\right)^{\frac{1}{D}-1}(1-D)}
$$

is called crucial constant.

By Theorem 1.1, the order of $k$ for the second term in (1.12) and (1.13) respectively will be the best. Now let us give more precise lower bound for the second term as follows.

Theorem 1.3 (Precise lower bounds). Under the conditions of Theorem 1.2, then for all integer $k \geq 1$ we have

$$
|\Omega| \frac{\sqrt{\lambda_{k}}}{\pi} \geq C_{k}
$$

where $C_{k}$ is the positive solution of equation

$$
x=k+\frac{P}{|\Omega|^{D}} x^{D}
$$

and $P$ is the same as Theorem 1.2. Moreover, for $k \geq 1$,

$$
C_{k} \geq k+\frac{P}{|\Omega|^{D}} k^{D}
$$

Observe that the estimate of the lower bounds in Theorem 1.3 is more precise than the result in Theorem 1.2.

Remark 1.1. 1. By Theorem 1.3, we have

$$
\lambda_{k} \geq \frac{\pi^{2} k^{2}}{|\Omega|^{2}}+\frac{2 \pi^{2} P}{|\Omega|^{D+2}} C_{k}^{D} k+\frac{\pi^{2} P^{2}}{|\Omega|^{2 D+2}} C_{k}^{2 D} .
$$

It is easy to check that $C_{k}^{D} k=\mathcal{O}\left(k^{1+D}\right)$, so the order of $k$ in the second term here is also the best. 
2. If there exists $L>0$ such that $\ell_{j} \sim L j^{-\frac{1}{D}}$ as $j \rightarrow+\infty$. Then the boundary $\Gamma$ is Minkowski measuable with Minkowski measure

$$
\mathcal{M}(D ; \Gamma)=\frac{2^{1-D}}{1-D} L^{D}
$$

Thus we can choose $\alpha_{*}=\gamma_{*} L, \alpha^{*}=\gamma^{*} L$ and $\ell_{1}=\gamma_{1} L$, then the crucial constant in Theorems 1.2 and 1.3 becomes

$$
P=P_{\mathcal{M}}=\frac{D \gamma_{*} \mathcal{M}(D ; \Gamma)}{\left(\gamma^{* D}+\gamma_{1}^{D}\right)^{\frac{1}{D}-1} 2^{1-D}} .
$$

In this paper, we will prove Theorem 1.1 in Section 2, and proofs of Theorems 1.2 and 1.3 will be given in Section 3. Finally, we shall give some examples in Section 4.

\section{Proof of Theorem 1.1}

\subsection{Proof of Theorem 1.1}

Proposition 2.1. Under the conditions of Theorem 1.1, if $\ell_{j} \asymp j^{-\frac{1}{D}}$ (as $j \rightarrow+\infty$ ) with $D \in$ $(0,1),\left\{\lambda_{k}\right\}_{k=1}^{\infty}$ is the Direchlet eigenvalues of $(1.1), N(\lambda)$ is counting function of $\left\{\lambda_{k}\right\}_{k=1}^{\infty}$, then

$$
\varphi(\lambda)-N(\lambda) \asymp \lambda^{\frac{D}{2}}, \quad \lambda \rightarrow+\infty,
$$

is equivalent to

$$
\lambda_{k}-\frac{\pi^{2}}{|\Omega|^{2}} k^{2} \asymp k^{1+D}, \quad k \rightarrow+\infty,
$$

where $\varphi(\lambda)=|\Omega| \frac{\sqrt{\lambda}}{\pi}$.

Proof. If (2.1) holds, then there exist $0<\delta_{*} \leq \delta^{*}<+\infty$ and $\lambda_{0}$ large enough, such that when $\lambda>\lambda_{0}$,

$$
\delta_{*} \lambda^{\frac{D}{2}} \leq \varphi(\lambda)-N(\lambda)=|\Omega| \frac{\sqrt{\lambda}}{\pi}-N(\lambda) \leq \delta^{*} \lambda^{\frac{D}{2}} .
$$

Then

$$
|\Omega| \frac{\sqrt{\lambda}}{\pi}-\delta^{*} \lambda^{\frac{D}{2}} \leq N(\lambda) \leq|\Omega| \frac{\sqrt{\lambda}}{\pi}-\delta_{*} \lambda^{\frac{D}{2}}
$$

Also we know that there exists $K_{1}>0$, such that when $k>K_{1}$, we have $\lambda_{k}>\lambda_{0}$. And it's obvious that $N\left(\lambda_{k}\right) \geq k$. Then

$$
k \leq N\left(\lambda_{k}\right) \leq|\Omega| \frac{\sqrt{\lambda_{k}}}{\pi}-\delta_{*} \lambda_{k}^{\frac{D}{2}}
$$


On the other hand, we notice that $N(\lambda)$ is right continuous step function. So we construct a left continuous step function $M(\lambda)$ when $\lambda>\lambda_{0}$ :

$$
M(\lambda)= \begin{cases}N(\lambda), & \lambda \text { is continuous point of } N(\lambda), \\ \lim _{p \rightarrow \lambda^{-}} N(p), & \text { anywhere else. }\end{cases}
$$

It is easy to obtain $M\left(\lambda_{k}\right)<k$ for all $\lambda_{k}>\lambda_{0}$. Now, if $\lambda>\lambda_{0}$, when $\lambda$ is continuous point of $N(\lambda)$,

$$
M(\lambda)=N(\lambda) \geq|\Omega| \frac{\sqrt{\lambda}}{\pi}-\delta^{*} \lambda^{\frac{D}{2}}
$$

when $\lambda$ is not continuous point of $N(\lambda)$,

$$
M(\lambda)=\lim _{p \rightarrow \lambda^{-}} N(p) \geq \lim _{p \rightarrow \lambda^{-}}\left(|\Omega| \frac{\sqrt{p}}{\pi}-\delta^{*} p^{\frac{D}{2}}\right)=|\Omega| \frac{\sqrt{\lambda}}{\pi}-\delta^{*} \lambda^{\frac{D}{2}}
$$

So for all $\lambda>\lambda_{0}$, we obtain

$$
M(\lambda) \geq|\Omega| \frac{\sqrt{\lambda}}{\pi}-\delta^{*} \lambda^{\frac{D}{2}}
$$

Furthermore, since

$$
\lambda_{k}^{\frac{D}{2}} \in o\left(\sqrt{\lambda_{k}}\right) \text { as } k \rightarrow \infty,
$$

then there exists $K_{2}>0$ such that

$$
|\Omega| \frac{\sqrt{\lambda_{k}}}{\pi}-\delta^{*} \lambda_{k}^{\frac{D}{2}}>0
$$

if $k \geq K_{2}$. Thus, when $k>\max \left\{K_{1}, K_{2}\right\}$,

$$
k>M\left(\lambda_{k}\right) \geq|\Omega| \frac{\sqrt{\lambda_{k}}}{\pi}-\delta^{*} \lambda_{k}^{\frac{D}{2}}>0 .
$$

Now we consider the following:

$$
b_{k}=: \frac{\lambda_{k}-\frac{\pi^{2} k^{2}}{|\Omega|^{2}}}{k^{1+D}}=\frac{|\Omega|^{2} \lambda_{k}-\pi^{2} k^{2}}{|\Omega|^{2} k^{1+D}} .
$$

By (2.4) and (2.6),

$$
\begin{aligned}
& b_{k} \leq \frac{|\Omega|^{2} \lambda_{k}-\pi^{2}\left(|\Omega| \frac{\sqrt{\lambda_{k}}}{\pi}-\delta^{*} \lambda_{k}^{\frac{D}{2}}\right)^{2}}{|\Omega|^{2}\left(|\Omega| \frac{\sqrt{\lambda_{k}}}{\pi}-\delta^{*} \lambda_{k}^{\frac{D}{2}}\right)^{1+D}}=\frac{2|\Omega| \pi \delta^{*} \lambda_{k}^{\frac{1+D}{2}}-\pi^{2} \delta^{* 2} \lambda_{k}^{D}}{|\Omega|^{2}\left(|\Omega| \frac{\sqrt{\lambda_{k}}}{\pi}-\delta^{*} \lambda_{k}^{\frac{D}{2}}\right)^{1+D}} \\
& b_{k} \geq \frac{|\Omega|^{2} \lambda_{k}-\pi^{2}\left(|\Omega| \frac{\sqrt{\lambda_{k}}}{\pi}-\delta_{*} \lambda_{k}^{\frac{D}{2}}\right)^{2}}{|\Omega|^{2}\left(|\Omega| \frac{\sqrt{\lambda_{k}}}{\pi}-\delta_{*} \lambda_{k}^{\frac{D}{2}}\right)^{1+D}}=\frac{2|\Omega| \pi \delta_{*} \lambda_{k}^{\frac{1+D}{2}}-\pi^{2} \delta_{*}^{2} \lambda_{k}^{D}}{|\Omega|^{2}\left(|\Omega| \frac{\sqrt{\lambda_{k}}}{\pi}-\delta_{*} \lambda_{k}^{\frac{D}{2}}\right)^{1+D}} .
\end{aligned}
$$


Let $k \rightarrow \infty$, since $1+D>2 D$, the right hand side of $(2.8 \mathrm{a})$ goes to $2\left(\frac{\pi}{|\Omega|}\right)^{2+D} \delta^{*}$ and the same of $(2.8 b)$ goes to $2\left(\frac{\pi}{|\Omega|}\right)^{2+D} \delta_{*}$. Thus we obtain (2.2).

Conversely, consider the interval $\left[\lambda_{k}, \lambda_{k+t}\right)$, where $\lambda_{k-1}<\lambda_{k}=\lambda_{k+1}=\cdots=$ $\lambda_{k+t-1}<\lambda_{k+t}$. Thus for any $\lambda \in\left[\lambda_{k}, \lambda_{k+t}\right), N(\lambda)=k+t-1$. So in $\left[\lambda_{k}, \lambda_{k+t}\right)$,

$$
f(\lambda)=: \frac{|\Omega| \frac{\sqrt{\lambda}}{\pi}-N(\lambda)}{\lambda^{\frac{D}{2}}}=\frac{|\Omega|}{\pi} \lambda^{\frac{1-D}{2}}-(k+t-1) \lambda^{-\frac{D}{2}}
$$

is monotonically increasing and continuous. We call every interval like $\left[\lambda_{k}, \lambda_{k+t}\right)$ a continuous and monotonic interval (CM interval) of $f(\lambda)$. Since $\lambda_{k}$ is finite multiplicity, every $\mathrm{CM}$ interval has finite length and there are infinitely many $\mathrm{CM}$ intervals.

If we take the subsequence $\left\{\lambda_{k_{j}}: N\left(\lambda_{k_{j}}\right)=k_{j}\right\}$, then $\left[\lambda_{k_{j}}, \lambda_{k_{j+1}}\right)$ turns out to be a CM interval of $f(x)$. Noticing that $\lambda_{k_{j+1}}=\lambda_{k_{j}+1}$, we rewrite $\left[\lambda_{k_{j}}, \lambda_{k_{j+1}}\right)$ as $\left[\lambda_{k_{j}}, \lambda_{k_{j}+1}\right)$. Hence for any $\lambda \in\left[\lambda_{k_{j}}, \lambda_{k_{j}+1}\right)$,

$$
f(\lambda)=\frac{|\Omega| \frac{\sqrt{\lambda}}{\pi}-N(\lambda)}{\lambda^{\frac{D}{2}}} \leq \frac{|\Omega| \frac{\sqrt{\lambda_{k_{j}+1}}}{\pi}-N\left(\lambda_{k_{j}}\right)}{\left(\sqrt{\lambda_{k_{j}+1}}\right)^{D}} \triangleq \theta_{j},
$$

where one should notice that

$$
\lim _{\lambda \rightarrow \lambda_{k_{j}+1}^{-}} N(\lambda)=N\left(\lambda_{k_{j}}\right)
$$

In fact,

$$
\theta_{j}=\sup _{\lambda \in\left[\lambda_{k_{j}}, \lambda_{k_{j}+1}\right)}\{f(\lambda)\}
$$

and

$$
\limsup _{\lambda \rightarrow+\infty} f(\lambda)=\lim _{j \rightarrow+\infty} \theta_{j}
$$

Since the condition (2.2) and the formula (2.7) give that

$$
\lambda_{k}-\frac{\pi^{2}}{|\Omega|^{2}} k^{2}=b_{k} k^{1+D} \asymp k^{1+D} \quad \text { as } k \rightarrow+\infty,
$$

then there exist $K>0$ and $0<b_{*} \leq b^{*}<+\infty$, such that when $k>K$, we have $b_{*} \leq b_{k} \leq$ $b^{*}$. Here we can assume that

$$
\limsup _{k \rightarrow+\infty} b_{k}=b^{*} \quad \text { and } \quad \liminf _{k \rightarrow+\infty} b_{k}=b_{*} .
$$

Putting $\lambda_{k}$ into $\theta_{j}$, one has

$$
\theta_{j}=\frac{\sqrt{\left(k_{j}+1\right)^{2}+\frac{|\Omega|^{2}}{\pi^{2}} b_{k_{j}+1}\left(k_{j}+1\right)^{1+D}}-k_{j}}{\left(\sqrt{\frac{\pi^{2}\left(k_{j}+1\right)^{2}}{|\Omega|^{2}}+\left(k_{j}+1\right)^{1+D} b_{k_{j}+1}}\right)^{D}}
$$




$$
=\frac{2 k_{j}+1+\frac{|\Omega|^{2}}{\pi^{2}} b_{k_{j}+1}\left(k_{j}+1\right)^{1+D}}{\left(\sqrt{\frac{\pi^{2}\left(k_{j}+1\right)^{2}}{|\Omega|^{2}}+\left(k_{j}+1\right)^{1+D} b_{k_{j}+1}}\right)^{D}\left(\sqrt{\left(k_{j}+1\right)^{2}+\frac{|\Omega|^{2}}{\pi^{2}} b_{k_{j}+1}\left(k_{j}+1\right)^{1+D}}+k_{j}\right)} .
$$

Since there are infinitely many CM intervals, then $j \rightarrow+\infty$ is equivalent to $k_{j} \rightarrow+\infty$. Hence

$$
\limsup _{j \rightarrow \infty} \theta_{j}=\frac{b^{*}}{2}\left(\frac{|\Omega|}{\pi}\right)^{D+2}
$$

By (2.10) and (2.13), we get

$$
\lim _{\lambda \rightarrow+\infty} f(\lambda) \leq \limsup _{\lambda \rightarrow+\infty} f(\lambda)=\lim _{j \rightarrow \infty} \theta_{j} \leq \limsup _{j \rightarrow \infty} \theta_{j}=\frac{b^{*}}{2}\left(\frac{|\Omega|}{\pi}\right)^{D+2}<+\infty .
$$

By using the same method, for any $\lambda \in\left[\lambda_{k_{j}}, \lambda_{k_{j}+1}\right)$, we have

$$
f(\lambda)=\frac{|\Omega| \frac{\sqrt{\lambda}}{\pi}-N(\lambda)}{\lambda^{\frac{D}{2}}} \geq \frac{|\Omega| \frac{\sqrt{\lambda_{k_{j}}}}{\pi}-N\left(\lambda_{k_{j}}\right)}{\left(\sqrt{\lambda_{k_{j}}}\right)^{D}} \triangleq \eta_{j} .
$$

Thus

$$
\eta_{j}=\inf _{\lambda \in\left[\lambda_{k_{j}}, \lambda_{k_{j}+1}\right)}\{f(\lambda)\}
$$

and

$$
\liminf _{\lambda \rightarrow+\infty} f(\lambda)=\lim _{j \rightarrow \infty} \eta_{j}
$$

Putting $\lambda_{k}$ into $\eta_{j}$, we obtain

$$
\eta_{j}=\frac{\frac{|\Omega|^{2}}{\pi^{2}} b_{k_{j}} k_{j}^{1+D}}{\left(\sqrt{\frac{\pi^{2} k_{j}^{2}}{|\Omega|^{2}}+k_{j}{ }^{1+D} b_{k_{j}}}\right)^{D}\left(\sqrt{k_{j}^{2}+\frac{|\Omega|^{2}}{\pi^{2}} b_{k_{j}} k_{j}^{1+D}}+k_{j}\right)} .
$$

Therefore

$$
\liminf _{j \rightarrow \infty} \eta_{j}=\frac{b_{*}}{2}\left(\frac{|\Omega|}{\pi}\right)^{D+2}
$$

So (2.15) and (2.16) imply

$$
\lim _{\lambda \rightarrow+\infty} f(\lambda) \geq \liminf _{\lambda \rightarrow+\infty} f(\lambda)=\lim _{j \rightarrow \infty} \eta_{j} \geq \liminf _{j \rightarrow \infty} \eta_{j}=\frac{b_{*}}{2}\left(\frac{|\Omega|}{\pi}\right)^{D+2}>0 .
$$

Consequently, by (2.14) and (2.17), we can deduce that (2.1). This completes the proof of Proposition 2.1. 
Next, we consider the case of $\ell_{j} \sim L j^{-\frac{1}{D}},(j \rightarrow \infty)$.

Proposition 2.2. Under the conditions of Theorem 1.1, if $\ell_{j} \sim L j^{-\frac{1}{D}},(j \rightarrow \infty)$ holds for some $L>0$ with $D \in(0,1),\left\{\lambda_{k}\right\}_{k=1}^{\infty}$ is the eigenvalues of $(1.1)$, and $N(\lambda)$ is counting function of $\left\{\lambda_{k}\right\}_{k=1}^{\infty}$. Then

$$
\varphi(\lambda)-N(\lambda) \sim \frac{-\zeta(D) L^{D}}{\pi^{D}} \lambda^{\frac{D}{2}} \text { for } \lambda \rightarrow+\infty,
$$

is equivalent to

$$
\lim _{k \rightarrow \infty} b_{k}=\frac{-2 \pi^{2} \zeta(D) L^{D}}{|\Omega|^{2+D}}
$$

where $b_{k}$ is defined as (2.7).

Proof. First, if (2.19) holds, then from (2.12) we have

$$
b_{*}=b^{*}=\frac{-2 \pi^{2} \zeta(D) L^{D}}{|\Omega|^{2+D}} .
$$

Thus

$$
\frac{-\zeta(D) L^{D}}{\pi^{D}}=\liminf _{j \rightarrow \infty} \eta_{j} \leq \lim _{\lambda \rightarrow \infty} f(\lambda) \leq \limsup _{j \rightarrow \infty} \theta_{j}=\frac{-\zeta(D) L^{D}}{\pi^{D}},
$$

where $f(\lambda)$ defined as (2.9). So we obtain (2.18).

Conversely, if (2.18) holds, by Proposition 2.1, $b_{k}$ will be bounded. Thus we only need to prove that

$$
b_{*}=b^{*}=\frac{-2 \pi^{2} \zeta(D) L^{D}}{|\Omega|^{2+D}} .
$$

We use the same method for $\lambda_{k_{j}}$ as that in Proposition 2.1. Then we also have the results in (2.13) and (2.16). It remains to prove

$$
\lim _{j \rightarrow+\infty} \eta_{j}=\lim _{j \rightarrow+\infty} \theta_{j}
$$

By (2.10) and (2.18), we obtain

$$
\lim _{j \rightarrow \infty} \theta_{j}=\limsup _{\lambda \rightarrow+\infty} f(\lambda)=\lim _{\lambda \rightarrow+\infty} f(\lambda)=\frac{-\zeta(D) L^{D}}{\pi^{D}},
$$

which, with (2.13), implies

$$
\frac{b^{*}}{2}\left(\frac{|\Omega|}{\pi}\right)^{D+2}=\limsup _{j \rightarrow \infty} \theta_{j}=\lim _{j \rightarrow \infty} \theta_{j}=\frac{-\zeta(D) L^{D}}{\pi^{D}} .
$$

Hence

$$
b^{*}=\frac{-2 \pi^{2} \zeta(D) L^{D}}{|\Omega|^{2+D}} .
$$


Next, we calculate $b_{*}$. By (2.15) and (2.18), we have

$$
\lim _{j \rightarrow \infty} \eta_{j}=\liminf _{\lambda \rightarrow+\infty} f(\lambda)=\lim _{\lambda \rightarrow+\infty} f(\lambda)=\frac{-\zeta(D) L^{D}}{\pi^{D}},
$$

which, with (2.16), implies

$$
\frac{b_{*}}{2}\left(\frac{|\Omega|}{\pi}\right)^{D+2}=\liminf _{j \rightarrow \infty} \eta_{j}=\lim _{j \rightarrow \infty} \eta_{j}=\frac{-\zeta(D) L^{D}}{\pi^{D}} .
$$

Hence

$$
b_{*}=\frac{-2 \pi^{2} \zeta(D) L^{D}}{|\Omega|^{2+D}} .
$$

Consequently, by (2.20) and (2.21) we have (2.19). Proposition 2.2 is proved.

Finally, from the results of Lapidus [9] (see (1.7) and (1.8) above), the proof of Theorem 1.1 will be given directly by the results in Proposition 2.1 and Proposition 2.2.

\subsection{Multiplicity estimate}

As a direct application of Theorem 1.1, we give an estimate of $a_{k} \triangleq N\left(\lambda_{k}\right)-k$, which imples the estimate of the multiplicity for the eigenvalues $\lambda_{k}$.

Proposition 2.3. Under the conditions of Theorem 1.1, $\left\{\lambda_{k}\right\}_{k=1}^{\infty}$ is the Dirichlet eigenvalues of (1.1). $N(\lambda)$ is counting function of $\left\{\lambda_{k}\right\}_{k=1}^{\infty}$ and let $a_{k} \triangleq N\left(\lambda_{k}\right)-k$. For $D \in(0,1)$ and $L>0$, if $\ell_{j} \sim L j^{-\frac{1}{D}},(j \rightarrow \infty)$, then

$$
a_{k}=o\left(k^{D}\right) \text { as } k \rightarrow+\infty .
$$

Proof. If $\ell_{j} \sim L j^{-\frac{1}{D}},(j \rightarrow \infty)$, then from Lapidus's result (1.8) and Proposition 2.2, we obtain (2.18) and

$$
b \triangleq: \lim _{k \rightarrow \infty} b_{k}=\frac{\mathcal{T}_{D} \mathcal{M}(D ; \Gamma)}{|\Omega|^{2+D}}=\frac{-2 \pi^{2} \zeta(D) L^{D}}{|\Omega|^{2+D}} .
$$

Then we rewrite $\lambda_{k}$ as (2.11) again. By Example 1.1 we can define a local counting function

$$
N\left(\lambda ; \Omega_{j}\right) \triangleq \sharp\left\{m \geq 1: \frac{\pi^{2} m^{2}}{\ell_{j}^{2}} \leq \lambda\right\}=\left[\ell_{j} \frac{\sqrt{\lambda}}{\pi}\right]
$$

for fixed $\Omega_{j}$, where $[x]$ means the largest integer which is not smaller than $x$. Then we have

$$
N(\lambda)=\sum_{j=1}^{\infty} N\left(\lambda ; \Omega_{j}\right)=\sum_{j=1}^{\infty}\left[\ell_{j} \frac{\sqrt{\lambda}}{\pi}\right] .
$$


Thus,

$$
\begin{aligned}
a_{k}+k & =N\left(\lambda_{k}\right)=\sum_{j=1}^{\infty}\left[\ell_{j} \sqrt{\frac{k^{2}}{|\Omega|^{2}}+\frac{b_{k}}{\pi^{2}} k^{1+D}}\right] \\
& \leq \sum_{j=1}^{\infty} \ell_{j} \sqrt{\frac{k^{2}}{|\Omega|^{2}}+\frac{b_{k}}{\pi^{2}} k^{1+D}} \\
& =\sqrt{k^{2}+\frac{|\Omega|^{2} b_{k}}{\pi^{2}} k^{1+D}} .
\end{aligned}
$$

Let $k \rightarrow+\infty$, we have

$$
\begin{aligned}
& 0 \leq \frac{a_{k}}{k} \leq \frac{\sqrt{k^{2}+\frac{|\Omega|^{2} b_{k}}{\pi^{2}} k^{1+D}}-k}{k} \rightarrow 0, \\
& 0 \leq \frac{a_{k}}{k^{D}} \leq \frac{\sqrt{k^{2}+\frac{|\Omega|^{2} b_{k}}{\pi^{2}} k^{1+D}}-k}{k^{D}} \rightarrow \frac{|\Omega|^{2} b}{2 \pi^{2}} .
\end{aligned}
$$

Hence $a_{k}=o(k)$ and $a_{k}=\mathcal{O}\left(k^{D}\right)$. More precise, we put $\lambda_{k}$ into $f(\lambda)$, then we have

$$
\begin{aligned}
f\left(\lambda_{k}\right) & =\frac{\sqrt{k^{2}+\frac{|\Omega|^{2}}{\pi^{2}} b_{k} k^{1+D}}-k-a_{k}}{\left(\sqrt{\frac{\pi^{2} k^{2}}{|\Omega|^{2}}+k^{1+D} b_{k}}\right)^{D}} \\
& =\frac{\frac{|\Omega|^{2}}{\pi^{2}} b_{k}-2 \frac{a_{k}}{k^{D}}-\frac{a_{k}^{2}}{k^{1+D}}}{\left(\sqrt{\frac{\pi^{2}}{|\Omega|^{2}}+k^{D-1} b_{k}}\right)^{D}\left(\sqrt{1+\frac{|\Omega|^{2}}{\pi^{2}} b_{k} k^{D-1}}+1+\frac{a_{k}}{k}\right.},
\end{aligned}
$$

which, with (2.18), (2.22), (2.24a) and (2.24b), implies

$$
\begin{aligned}
& \lim _{k \rightarrow \infty}\left(\frac{|\Omega|^{2}}{\pi^{2}} b_{k}-2 \frac{a_{k}}{k^{D}}\right) \\
= & \lim _{k \rightarrow \infty}\left(f\left(\lambda_{k}\right)\left(\sqrt{\frac{\pi^{2}}{|\Omega|^{2}}+k^{D-1} b_{k}}\right)^{D}\left(\sqrt{1+\frac{|\Omega|^{2}}{\pi^{2}} b_{k} k^{D-1}}+1+\frac{a_{k}}{k}\right)+\frac{a_{k}^{2}}{k^{1+D}}\right) \\
= & \frac{-2 \zeta(D) L^{D}}{|\Omega|^{D}} .
\end{aligned}
$$

Combining with (2.19), we obtain that

$$
\lim _{k \rightarrow \infty} 2 \frac{a_{k}}{k^{D}}=0,
$$

which means $a_{k}=o\left(k^{D}\right)$ as $k \rightarrow+\infty$. Proposition 2.3 is proved. 
There are a lot of examples in which $a_{k} \rightarrow+\infty$ as $k \rightarrow+\infty$ (e.g., $\Omega \subset \mathbb{R}$ is a Cantor string). However, here we can give a special example in which for each $k$, we have $a_{k}=0$.

Example 2.1. Let $\Omega=\bigcup_{j=1}^{\infty} \Omega_{j}$, with $\left|\Omega_{j}\right|=\ell_{j}=j^{-\sqrt{2}}$. Then Minkowski dimension of $\partial \Omega$ is $D=\frac{1}{\sqrt{2}} \in(0,1)$. In this case we have $a_{k}=0$ for each $k$.

Proof. Assume that the multiplicity of eigenvalue $\lambda_{k}$ is large than 1 , then there exist $k_{1}, k_{2}, j_{1}, j_{2} \in \mathbb{N} \backslash\{0\}$, such that

$$
\pi^{2} k_{1}^{2} j_{1}^{2 \sqrt{2}}=\pi^{2} k_{2}^{2} j_{2}^{2 \sqrt{2}} \text { and }\left(k_{1}-k_{2}\right)\left(j_{1}-j_{2}\right) \neq 0 .
$$

That implies

$$
\frac{k_{1}}{k_{2}}=\left(\frac{j_{2}}{j_{1}}\right)^{\sqrt{2}} \text {. }
$$

This is a contradiction with Gelfond-Schneider Theorem (see [1,6]) that says if $r$ and $t$ are algebraic numbers with $r \neq 0,1$, and $t$ is irrational, then $r^{t}$ is a transcendental number. Thus the multiplicity for each eigenvalue is 1 , that means $a_{k}=N\left(\lambda_{k}\right)-k=0$.

\section{Proof of Theorem 1.2 and Theorem 1.3}

\subsection{Proof of Theorem 1.2}

First, from (2.23) we have

$$
k \leq N\left(\lambda_{k}\right)=\sum_{j=1}^{\infty}\left[\ell_{j} \frac{\sqrt{\lambda_{k}}}{\pi}\right] \leq \sum_{j=1}^{\infty} \ell_{j} \frac{\sqrt{\lambda_{k}}}{\pi}=|\Omega| \frac{\sqrt{\lambda_{k}}}{\pi} .
$$

Thus,

$$
\begin{aligned}
& \lambda_{k} \geq \frac{\pi^{2}}{|\Omega|^{2}} k^{2}, \\
& k \leq N\left(\lambda_{k}\right)=\sum_{j=1}^{\infty}\left[\ell_{j} \frac{\sqrt{\lambda_{k}}}{\pi}\right]=\sum_{j=1}^{\infty} \ell_{j} \frac{\sqrt{\lambda_{k}}}{\pi}-\sum_{j=1}^{\infty}\left\{\ell_{j} \frac{\sqrt{\lambda_{k}}}{\pi}\right\},
\end{aligned}
$$

where $\{x\}=x-[x] \in[0,1)$. Let $x=\frac{\sqrt{\lambda_{k}}}{\pi}$, then the condition (1.11) gives that when $j>\left(\alpha^{*} x\right)^{D}, \ell_{j} x<1$ holds. So $\left\{\ell_{j} x\right\}=\ell_{j} x$. Hence, in the case of $\ell_{j} \geq \alpha_{*} j^{-\frac{1}{D}}$, one has

$$
\begin{aligned}
\sum_{j=1}^{\infty}\left\{\ell_{j} x\right\} & =\sum_{j \leq\left(\alpha^{*} x\right)^{D}}\left\{\ell_{j} x\right\}+\sum_{j>\left(\alpha^{*} x\right)^{D}} \ell_{j} x \\
& \geq \alpha_{*} x \sum_{j \geq\left[\left(\alpha^{*} x\right)^{D}\right]+1} j^{-\frac{1}{D}} \\
& \geq \frac{x D \alpha_{*}}{(1-D)\left(\left[\left(\alpha^{*} x\right)^{D}\right]+1\right)^{\frac{1}{D}-1}} .
\end{aligned}
$$


Observe that $x \geq \frac{\sqrt{\lambda_{1}}}{\pi}=\frac{1}{\ell_{1}}$, we have

$$
\left[\left(\alpha^{*} x\right)^{D}\right]+1 \leq\left(\alpha^{*} x\right)^{D}+1 \leq\left(\alpha^{* D}+\ell_{1}^{D}\right) x^{D} .
$$

Then

$$
\left(\left[\left(\alpha^{*} x\right)^{D}\right]+1\right)^{\frac{1}{D}-1} \leq\left(\alpha^{* D}+\ell_{1}^{D}\right)^{\frac{1}{D}-1} x^{1-D} .
$$

Thus from (3.2) one has

$$
\sum_{j=1}^{\infty}\left\{\ell_{j} x\right\} \geq \frac{x D \alpha_{*}}{(1-D)\left(\left[\left(\alpha^{*} x\right)^{D}\right]+1\right)^{\frac{1}{D}-1}} \geq \frac{x D \alpha_{*}}{\left(\alpha^{* D}+\ell_{1}^{D}\right)^{\frac{1}{D}-1}(1-D) x^{1-D}}=P x^{D},
$$

where

$$
P=\frac{D \alpha_{*}}{\left(\alpha^{* D}+\ell_{1}^{D}\right)^{\frac{1}{D}-1}(1-D)} .
$$

Putting (3.5) into (3.1b), we have

$$
k \leq \sum_{j=1}^{\infty} \ell_{j} \frac{\sqrt{\lambda_{k}}}{\pi}-\sum_{j=1}^{\infty}\left\{\ell_{j} \frac{\sqrt{\lambda_{k}}}{\pi}\right\} \leq|\Omega| \frac{\sqrt{\lambda_{k}}}{\pi}-P\left(\frac{\sqrt{\lambda_{k}}}{\pi}\right)^{D} .
$$

Thus, from (3.1a), one has

$$
|\Omega| \frac{\sqrt{\lambda_{k}}}{\pi} \geq k+P\left(\frac{\sqrt{\lambda_{k}}}{\pi}\right)^{D} \geq k+\frac{P}{|\Omega|^{D}} k^{D} .
$$

The estimate (1.12) is proved. That means

$$
\lambda_{k} \geq \frac{\pi^{2}}{|\Omega|^{2}} k^{2}+\frac{2 \pi^{2} P}{|\Omega|^{2+D}} k^{1+D}+\frac{\pi^{2} P^{2}}{|\Omega|^{2+2 D}} k^{2 D} .
$$

Theorem 1.2 is proved.

Corollary 3.1. Under the conditions of Theorem 1.2, if $\mathcal{L}=\left\{\ell_{j}\right\}_{j=1}^{\infty}$ is Minkowski measurable, that is, $\ell_{j} \sim L j^{-\frac{1}{D}},(j \rightarrow \infty)$, then (1.13) holds and the crucial constant P becomes

$$
P=P_{\mathcal{M}}=\frac{D \mu_{*} \mathcal{M}(D ; \Gamma)}{\left(\mu_{1}^{D}+\mu^{* D}\right)^{\frac{1}{D}-1} 2^{1-D}}
$$

where $\alpha^{*}=\mu^{*} L, \alpha_{*}=\mu_{*} L, \ell_{1}=\mu_{1} L$ and $\mathcal{N}(D ; \Gamma)=\frac{2^{1-D} L^{D}}{1-D}$. Meanwhile, Theorem 1.3 has a similar corollary. 


\subsection{Proof of Theorem 1.3}

Lemma 3.1. We have the following results:

1. There is only one positive root of the equation $x=k+B x^{D}$, where $0<D<1, k \geq 1$ and $B>0$.

2. Denote the positive root by $x_{0}$. For any $x_{1}>0$, we set a sequence $\left\{x_{n}=: x_{n}\left(x_{1}\right)\right\}_{n=1}^{\infty}$ as defined by $x_{n+1}=k+B x_{n}^{D}$ for $n \geq 1$. Then

$$
\lim _{n \rightarrow+\infty} x_{n}=x_{0}
$$

That means, for any $x_{1}>0, x_{n}$ has the same limitation $x_{0}$.

Proof. Let $s(x)=k+B x^{D}-x$, then $s(0)=k>0$ and $s(x) \rightarrow-\infty$ as $x \rightarrow+\infty$. So $s(x)$ has at least a root in $(0,+\infty)$. Next we show the positive root is unique. Noticing that $s^{\prime}(x)=D B x^{D-1}-1$, and we have $s(x)$ is monotonically increasing in $\left(0,(D B)^{\frac{1}{1-D}}\right)$ and monotonically decreasing in $\left((D B)^{\frac{1}{1-D}},+\infty\right)$. Also $s(x)>0$ in $\left(0,(D B)^{\frac{1}{1-D}}\right)$ for $s(0)=$ $k>0$. So the zeros must be in $\left((D B)^{\frac{1}{1-D}},+\infty\right)$ and there is only one root. Now we have proved the first part of the lemma. We denote the root by $x_{0}$ and $s(x)>0$ in $\left(0, x_{0}\right)$. To the second part of this lemma. If $x_{1}=x_{0}$, then $x_{n}=x_{0}$ and the limit is $x_{0}$. If $0<x_{1}<x_{0}$, by induction we can easily prove that for any $n \geq 1, x_{n}<x_{0}$. Next, $x_{n+1}-x_{n}=k+B x_{n}^{D}-x_{n}>0$ since $x_{n}<x_{0}$ and $s(x)>0$ in $\left(0, x_{0}\right)$. Thus $\left\{x_{n}\right\}$ is monotonically increasing. Hence $x_{n}$ has the limitation that is the positive solution of equation $x=k+B x^{D}$. The proof for $x_{1}>x_{0}$ will be similar so we omit the details. Hence we have proved the equation $x=k+B x^{D}$ has only one positive root, Lemma 3.1 is proved.

Now we give the proof of Theorem 1.3.

Proof. By (3.6), we have

$$
|\Omega| \frac{\sqrt{\lambda_{k}}}{\pi} \geq k+P\left(\frac{\sqrt{\lambda_{k}}}{\pi}\right)^{D}
$$

Let $T_{k}=|\Omega| \frac{\sqrt{\lambda_{k}}}{\pi}$, then (3.7) becomes

$$
T_{k} \geq k+\frac{P}{|\Omega|^{D}} T_{k}^{D}
$$

Consider the sequence $\left\{x_{n}(k)\right\}_{n=1}^{\infty}$ :

$$
x_{1}(k)=k+\frac{P}{|\Omega|^{D}} T_{k}^{D} \quad \text { and } \quad x_{n+1}(k)=k+\frac{P}{|\Omega|^{D}}\left(x_{n}(k)\right)^{D} .
$$


Thus by induction we have for any fixed $k \geq 1, x_{n+1}(k) \leq x_{n}(k)$ holds for every $n \geq 1$. Thus, by iteration we have for $n \geq 1$

$$
T_{k} \geq x_{1}(k) \geq \cdots \geq x_{n}(k)
$$

Let $n \rightarrow+\infty$ and from Lemma 3.1, we have

$$
T_{k} \geq C_{k}
$$

where $C_{k}$ is the positive root of equation

$$
x=k+\frac{P}{|\Omega|^{D}} x^{D} .
$$

The estimate (1.15) in Theorem 1.3 is proved.

Next, we can prove that as the lower bound, $C_{k}$ is better than (1.12). Consider the sequence $\left\{y_{n}(k)\right\}_{n=1}^{\infty}$ :

$$
y_{1}(k)=k+\frac{P}{|\Omega|^{D}} k^{D} \quad \text { and } \quad y_{n+1}(k)=k+\frac{P}{|\Omega|^{D}}\left(y_{n}(k)\right)^{D} .
$$

Then from Lemma 3.1, if $n \rightarrow+\infty, y_{n}(k)$ has the same limitation $C_{k}$ as $x_{n}(k)$. Furthermore, observe that for each $k \geq 1$, we have $y_{n}(k) \leq y_{n+1}(k)$ for all $n \geq 1$, so

$$
C_{k} \geq y_{1}(k)=k+\frac{P}{|\Omega|^{D}} k^{D} .
$$

The estimate (1.16) holds and the proof of Theorem 1.3 is completed.

When $D=\frac{1}{2}, \frac{1}{3}, \frac{1}{4}$, one may solve the equation

$$
x=k+\frac{P}{|\Omega|^{D}} x^{D}
$$

by formula and we give the following result in the case of $D=\frac{1}{2}$.

Corollary 3.2. Under the conditions of Theorem 1.3, if $D=\frac{1}{2}$, then we have the sharp lower bound:

$$
|\Omega| \frac{\sqrt{\lambda_{k}}}{\pi} \geq k+\frac{P}{2|\Omega|} \sqrt{\left(4|\Omega| k+P^{2}\right)}+\frac{P^{2}}{2|\Omega|} .
$$

Proof. It is obvious from Theorem 1.3. 


\section{Examples}

In this section, we will apply our results to give the following examples. Here, if Minkowski dimension $D=\frac{1}{2}$, we will use Theorem 1.3, otherwise we use Theorem 1.2. First, we consider a usual example: $\ell_{j}=j^{-2}$. Then the Minkowski dimension of the boundary is $D=\frac{1}{2}$ and we have following example.

Example 4.1. Let the fractal string

$$
\Omega=\bigcup_{j=1}^{\infty} \Omega_{j} \subseteq \mathbb{R} \text { with }\left|\Omega_{j}\right|=\ell_{j}=j^{-2},
$$

then

$$
|\Omega|=\sum_{i=0}^{\infty} j^{-2}=\zeta(2)=\frac{\pi^{2}}{6}, \quad D=\frac{1}{2} \quad \text { and } \quad \mathcal{M}\left(\frac{1}{2} ; \Gamma\right)=2 \sqrt{2} .
$$

From Theorem 1.1, the Dirichelt eigenvalues of (1.1) have the following asymptotic expansion:

$$
\lambda_{k}=\frac{36}{\pi^{2}} k^{2}-2 \frac{6^{\frac{5}{2}} \zeta\left(\frac{1}{2}\right)}{\pi^{3}} k^{\frac{3}{2}}+o\left(k^{\frac{3}{2}}\right) \quad \text { as } k \rightarrow+\infty .
$$

On the other hand, from Theorem 1.3, for $k \geq 1$ we have

$$
\frac{\pi \sqrt{\lambda_{k}}}{6} \geq k+\frac{3}{2 \pi^{2}} \sqrt{\frac{2 \pi^{2}}{3} k+\frac{1}{4}}+\frac{3}{4 \pi^{2}} .
$$

The second example is called Cantor string which is defined as

$$
\mathfrak{C}=\left\{\ell_{j}\right\}_{j=1}^{\infty}=\left\{3^{\left[-\log _{2} j\right]}\right\}_{j=1}^{\infty}=\{\frac{1}{3^{2}}, \frac{1}{3^{2}}, \frac{1}{3^{2}}, \underbrace{\frac{1}{3^{3}}, \cdots, \frac{1}{3^{3}}}_{2^{2}}, \cdots\} .
$$

Then we have

Example 4.2. Let Cantor string $\mathfrak{C}$ be the fractal string of $\Omega \subseteq \mathbb{R}$. Then the Minkowski dimension

$$
D=\frac{\ln 2}{\ln 3} \quad \text { and } \quad|\Omega|=\sum_{i=0}^{\infty} \frac{2^{i}}{3^{i+1}}=1 .
$$

From Theorem 1.2, we take $\alpha_{*}=\frac{1}{3}$ and $\alpha^{*}=1$, the eigenvalues of (1.1) have the following lower bounds for all $k \geq 1$ :

$$
\lambda_{k} \geq \pi^{2} k^{2}+\frac{2 \pi^{2} \ln 2}{2 \cdot 3^{\frac{\ln 3}{\ln 2}-1}(\ln 3-\ln 2)} k^{1+\frac{\ln 2}{\ln 3}}+\pi^{2}\left(\frac{\ln 2}{2 \cdot 3^{\frac{\ln 3}{\ln 2}-1}(\ln 3-\ln 2)}\right)^{2} k^{2 \frac{\ln 2}{\ln 3}} .
$$


The last example is a fractal-string as defined by

$$
\mathfrak{A}=\left\{\ell_{j}\right\}_{j=1}^{\infty}=\left\{\frac{1}{j^{a}}-\frac{1}{(j+1)^{a}}\right\}_{j=1}^{\infty} .
$$

Then

$$
\ell_{j}=\frac{1}{j^{a}}-\frac{1}{(j+1)^{a}} \sim a j^{-a-1} \quad \text { as } j \rightarrow+\infty, \quad|\Omega|=\sum_{i=0}^{\infty} \ell_{j}=1,
$$

and the Minkowski dimension $D=\frac{1}{1+a}, \ell_{1} \leq L_{j} \leq a$. Thus we have

Example 4.3. $\mathfrak{A}$ is a fractal-string in $\mathbb{R}$ with Minkowski dimension $D=\frac{1}{1+a}$, which is Minkowski measurable with

$$
\mathcal{M}(D ; \Gamma)=\frac{2^{1-D} a^{D}}{1-D}=(a+1)\left(\frac{2}{a}\right)^{\frac{a}{a+1}} .
$$

Then from Theorem 1.1 the Dirichlet eigenvalues of (1.1) have the following asymptotic expansion:

$$
\lambda_{k}=\pi^{2} k^{2}-2 \pi^{2} \zeta\left(\frac{1}{a+1}\right) a^{\frac{1}{a+1}} k^{\frac{a+2}{a+1}}+o\left(k^{\frac{a+2}{a+1}}\right) \quad \text { as } \quad k \rightarrow+\infty .
$$

Meanwhile, from Theorem 1.2, the Dirichlet eigenvalues have the following lower bounds for $k \geq 1$ :

$$
\lambda_{k} \geq \pi^{2} k^{2}+\frac{2 \pi^{2} \ell_{1}}{a\left(a^{\frac{1}{1+a}}+\ell_{1}^{\frac{1}{1+a}}\right)^{a}} k^{\frac{a}{1+a}}+\frac{\pi^{2} \ell_{1}^{2}}{a^{2}\left(a^{\frac{1}{1+a}}+\ell_{1}^{\frac{1}{1+a}}\right)^{2 a}} k^{\frac{2 a}{1+a}},
$$

where $\ell_{1}=1-\frac{1}{2^{a}}$.

\section{Acknowledgements}

The research has been supported by the grants from National Natural Science Foundation of China (Grants No. 11631011).

\section{References}

[1] A. Baker, Transcendental Number Theory, Cambridge University Press, 1990.

[2] H. Chen, and B. D. Sleeman, Fractal drums and the $n$-dimensional modified Weyl-Berry conjecture, Commun. Math. Phys., 168(3) (1995), 581-607.

[3] H. Chen, and B. D. Sleeman, Counting function asymptotics and the weak Weyl-Berry conjecture for connected domains with fractal boundaries, Acta Math. Sinica, 14(2) (1998), 261276.

[4] L. C. Evans, Partial Differential Equations: Second Edition, American Mathmatical Society. 
[5] J. Fleckinger-Pellé, and D. G. Vassiliev, An example of a two term asymptotics for the "countingfunction" of a fractal drum, Trans. Am. Math. Soc., 337(1) (1993), 99-116.

[6] A. O. Gelfond, Transcendental and Algebraic Numbers, Courier Dover Publications, 2015.

[7] V. Y. Ivrii, Second term of the spectral asymptotic expansion of the Laplace-Beltrami operator on manifolds with boundary, Functional Anal. Appl., 14(2) (1980), 98-106.

[8] M. Kac, Can one hear the shape of a drum?, The American Mathematical Monthly, 73(4P2) (1966), 1-23.

[9] M. L. Lapidus, The Riemann Zeta-function and the one-dimensional Weyl-Berry conjecture for fractal drums, Proceedings of the London Mathematical Society, 3(1) (1993), 41-69.

[10] M. L. Lapidus, Spectral and fractal geometry: From the Weyl-Berry conjecture for the vibrations of fractal drums to the Riemann Zeta-function, Mathematics in Science and Engineering, Elsevier, 186 (1992), 151-181.

[11] M. L. Lapidus, and H. Maier, Hypothèse de Riemann, cordes fractales vibrantes et conjecture de Weyl-Berry modifiée, Comptes Rendus de l'Académie des Sciences, Série 1, Mathématique, 313(1) (1991), 19-24.

[12] P. Li and S. T. Yau, On the Schrödinger equation and the eigenvalue problem, Commun. Math. Phys., 88(3) (1983), 309-318.

[13] R. B. Melrose, Weyl's conjecture for manifolds with concave boundary, Proc. Sympos. Pure Math., 36 (1980), 257-274.

[14] G. Pólya, On the Eigenvalues of Vibrating Membranest: (In Memoriam Hermann Weyl) Proceedings of the London Mathematical Society, 3(1) (1961), 419-433.

[15] R. Seeley, A sharp asymptotic remainder estimate for the eigenvalues of the Laplacian in a domain of $\mathbb{R}^{3}$, Adv. Math., 29(2) (1978), 244-269.

[16] H. Weyl, Ramifications, old and new, of the eigenvalue problem, Bulletin of the American Mathematical Society, 56(2) (1950), 115-139.

[17] H. Weyl, Das asymptotische verteilungsgesetz der eigenwerte linearer partieller differentialgleichungen (mit einer anwendung auf die theorie der hohlraumstrahlung), Mathematische Annalen, 71(4) (1912), 441-479. 